\section{Real-Time-Glukosemessung ab 1. April berechnungsfähig}

_ Insulinpflichtige Diabetiker haben nach einem Beschluss des Bewertungsausschusses ab dem 8. März 2017 unter bestimmten Voraussetzungen Anspruch auf ein Real-Time-Messgerät zur kontinuierlichen interstitiellen Glukosemessung (rtCGM). Für die Anleitung des Patienten bzw. der Bezugsperson zur Selbstanwendung des Geräts werden am 1. April 2017 die Nrn. 03 355, 04590 und 13360 in den EBM aufgenommen. Die Beschränkung auf diese EBM-Kapitel er-

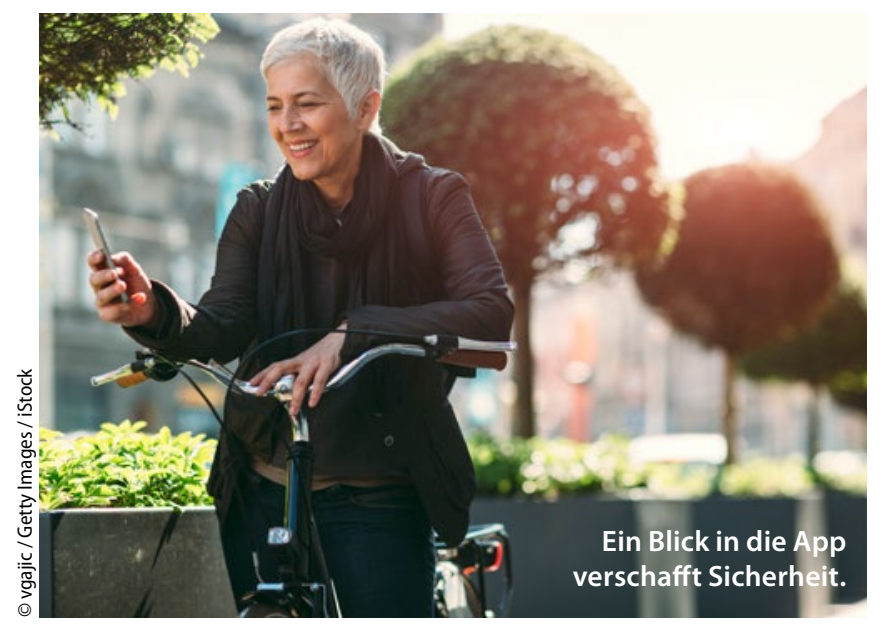

gibt sich aus der zugrunde liegenden Richtlinie „Methoden vertragsärztlicher Versorgung" (MVV-RL), in der der Gemeinsame Bundesausschuss (G-BA) u.a. die Fachgruppen definiert, die die Leistung erbringen dürfen.

Die drei Nrn. sind inhaltsgleich. Sie werden je vollendete zehn Minuten Unterweisungszeit abgerechnet und sind mit 72 Punkten bewertet. Im Krankheitsfall, also binnen vier Quartalen, können sie bis zu zehnmal angesetzt werden. Die Prüfzeit wurde auf nur zwei Minuten festgelegt, da die Anleitung entsprechend den Vorgaben der MVVRL auch teilweise an Praxispersonal delegiert werden kann.

\section{MMW-KOMMENTAR} Bereits am 16. Juni 2016 hatte der G-BA die rtCGM als anerkannte Untersuchungsmethode in der Therapiesteuerung bei insulinpflichtigen Diabetikern in

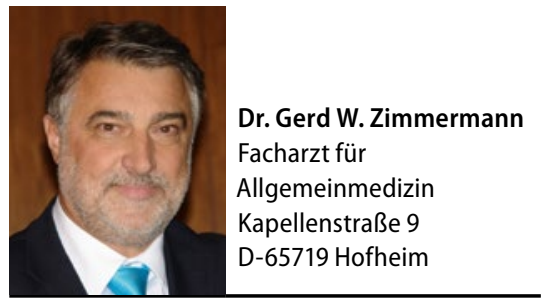

die Anlage I der MVV-RL aufgenommen. Hier sind auch die Indikationen und Vorgaben zur Qualitätssicherung geregelt. Dieser G-BABeschluss trat am 7. September 2016 in Kraft, sodass der Bewertungsausschuss den EBM aufgrund der Sechs-Monats-Frist gemäß § 87 Abs. 5b SGB V spätestens bis zum 7. März 2017 anpassen musste. Obwohl die EBMÄnderungen erst zum 1. April 2017 in Kraft treten, haben Patienten also bereits in der Zeit vom 8.-31. März 2017 Anspruch auf Kostenerstattung gegenüber ihrer Kasse. Die rtCGM durchführen und abrechnen können Fachärzte für Innere Medizin und Endokrinologie und Diabetologie. Daneben ist das auch Fachärzten für Innere Medizin, für Allgemeinmedizin oder für Kinder- und Jugendmedizin möglich, wenn sie die Anerkennung "Diabetologie" oder "Diabetologe $D D G$ " der Deutschen Diabetes Gesellschaft bzw. eine vergleichbare Qualifikation vorweisen können. Für Pädiater gibt es zudem die Anerkennung "Kinder-Endokrinologie und-Diabetologie".

Die neuen Leistungen werden zunächst außerhalb der morbiditätsbedingten Gesamtvergütungen und damit extrabudgetär vergütet.

\title{
Kosten für Website von der Steuer absetzen
}

_ Trotz des gelockerten Werbeverbots für Ärzte bleibt anpreisende Werbung untersagt - insbesondere auf der eigenen Internetseite. Erlaubt sind Visitenkarten am Empfang, Flyer mit Leistungen und Besonderheiten der Praxis, Patientenbroschüren mit allen wichtigen Informationen über die Praxis, aber auch Werbung über Radio, Regionalfernsehen und das Internet.

\section{MMW-KOMMENTAR}

Das alles kostet natürlich auch Geld. Derartige Ausgaben für Werbeaktionen können aber als Betriebsausgaben geltend gemacht werden. Das gilt z. B. für Zeitungsannoncen, Mailings aller Art und die Teilnahme an Messen, aber auch für die dazugehörigen Ausgaben etwa für eine Werbeagentur oder einen Grafiker. Auch Werbegeschenke als Streuartikel mit einem Einzelwert bis zu 10 Euro kön- nen abgesetzt werden. Und nicht zuletzt können die laufenden Kosten für den Internetauftritt als Betriebsausgaben geltend gemacht werden. Dazu zählen z. B. die Gebühren des Internetproviders für die Domain. Die Internetseite selbst gilt allerdings als immaterielles Wirtschaftsgut. Deren Kosten können deshalb nicht sofort geltend gemacht werden, sondern müssen als Anschaffungskosten abgeschrieben werden. 